\title{
Apatinib in treating patients with recurrent or metastatic nasopharyngeal carcinoma who had failed prior platinum-based chemotherapy
}

\author{
Chang-Juan Tao ${ }^{1 \#}$, Peng Zhang ${ }^{2 \#}$, Ling Zhou ${ }^{3 \#}$, Qiao-Ying Hu ${ }^{1}$, Xiao-Zhong Chen ${ }^{1}$ \\ ${ }^{1}$ Department of Radiation Oncology, Cancer Hospital of the University of Chinese Academy of Sciences (Zhejiang Cancer Hospital), Institute of \\ Cancer and Basic Medicine (IBMC), Chinese Academy of Sciences, Hangzhou, China; ${ }^{2}$ Department of Medical oncology, Cancer Hospital of the \\ University of Chinese Academy of Sciences (Zhejiang Cancer Hospital), Institute of Cancer and Basic Medicine (IBMC), Chinese Academy of \\ Sciences, Hangzhou, China; ${ }^{3}$ Graduate School, Guangdong Medical University, Zhanjiang, China \\ Contributions: (I) Conception and design: QY Hu, XZ Chen; (II) Administrative support: QY Hu, XZ Chen; (III) Provision of study materials or \\ patients: QY Hu, XZ Chen; (IV) Collection and assembly of data: CJ Tao, P Zhang, L Zhou; (V) Data analysis and interpretation: CJ Tao, P Zhang, \\ L Zhou; (VI) Manuscript writing: All authors; (VII) Final approval of manuscript: All authors. \\ \#These authors contributed equally to this work. \\ Correspondence to: Xiao-Zhong Chen. Department of Radiation Oncology, Institute of Cancer Research and Basic Medical Sciences of Chinese \\ Academy of Sciences, Cancer Hospital of University of Chinese Academy of Sciences, Zhejiang Cancer Hospital, Hangzhou, China. \\ Email: chenxiaozhongzjrt@163.com.
}

Background: Platinum-based chemotherapy is the standard first-line treatment for recurrent/metastatic nasopharyngeal carcinoma (NPC); however, there is no standard regimen for those who failed first-line treatment. This study aimed to evaluate the efficacy and safety of apatinib in treating patients with recurrent/ metastatic NPC who failed prior platinum-based chemotherapy.

Methods: Patients aged 18-65 years with recurrent/metastatic NPC were treated with apatinib at an initial dose of $500 \mathrm{mg}$ once daily and continued until disease progression, patient withdrawal, or unacceptable toxic effects. The primary endpoints were clinical benefit rate (CBR) and toxicity. Secondary endpoints included progression-free survival (PFS) and overall survival (OS).

Results: Nineteen patients were enrolled in this study. At the final follow-up, the CBR was $52.6 \%$ (95\% CI: 29.8-76.2\%) in the intention-to-treat population. The median PFS and OS were 3.7 (95\% CI: 0.6-6.8) months and 12.9 (95\% CI: 9.3-16.5) months, respectively. The most common grade 3-4 adverse events (AEs) were hand-foot syndrome [3 (15.8\%)], neutropenia [2 (10.5\%)], proteinuria [2 (10.5\%)], oral mucosal pain [2 (10.5\%)], hypertension [1 (5.3\%)], hyponatremia [1 (5.3\%)], artery dissection [1 (5.3\%)], and nasopharyngeal hemorrhage [1 (5.3\%)]. A serious AEs was reported in one patient who died of nasopharyngeal hemorrhage. Treatment with apatinib did not significantly influence patientreported quality-of-life, except for nausea/vomiting and pain $(\mathrm{P}<0.05)$.

Conclusions: Apatinib achieved modest disease control with acceptable toxicity in recurrent/metastatic NPC patients pretreated with platinum-based chemotherapy.

Keywords: Apatinib; nasopharyngeal carcinoma (NPC); recurrent or metastatic; platinum-based chemotherapy; toxicity

Submitted Jan 10, 2020. Accepted for publication Jan 30, 2020.

doi: $10.21037 /$ tcr-20-1773

View this article at: http://dx.doi.org/10.21037/tcr-20-1773 


\section{Introduction}

Nasopharyngeal carcinoma (NPC) is a common type of malignancy in south China and southeastern Asia $(1,2)$. The widespread application of intensity-modulated radiotherapy (IMRT) and an optimized chemotherapy regimen (induction, concurrent and adjuvant) have improved the 5 -year overall survival (OS) rates for NPC to about $85 \%$ (3). However, about $30 \%$ of NPC patients have recurrent or metastatic disease despite undergoing radical chemoradiotherapy (4).

Platinum-based chemotherapy is commonly used as a standard first-line treatment for NPC. A recently published phase III clinical study showed gemcitabine plus cisplatin exhibits preferable anti-tumor activity than fluorouracil plus cisplatin in recurrent or metastatic NPC [median progression-free survival (PFS) of 7.0 versus 5.6 months, respectively] (5). However, the optimal therapeutic regimen for those who failed this first-line chemotherapy have not yet been established.

Several multi-kinase tyrosine kinase inhibitors (e.g., sorafenib, sunitinib, pazopanib, and axitinib) have already shown a favorable clinical benefit in recurrent/metastatic NPC, with a median PFS of 3.2-7.2 months (6-9). However, a phase II study detected a high incidence of hemorrhagic complications with sunitinib in recurrent/metastatic NPC (9). Therefore, we require more effective and safe treatment regimens for patients with metastatic/recurrent NPC.

The vascular endothelial growth factor receptor (VEGFR) family of proteins consists of VEGFR-1, VEGFR-2, and VEGFR-3. Among them, VEGFR-2 is the principal mediator of VEGF-induced angiogenic signaling. Apatinib is a highly-selective inhibitor of VEGFR-2. Using an in vivo murine model of NPC, Peng et al. confirmed that the administration of apatinib inhibited tumor growth, reduced microvascular density, and facilitated tumor apoptosis in tumor-bearing mice (10). However, the clinical efficacy of apatinib in NPC patients remains unclear.

This phase II clinical study aimed to evaluate the efficacy and safety of apatinib in treating patients with recurrent/metastatic NPC who failed prior platinumbased chemotherapy. We also evaluated the quality-oflife (QOL) after administration of apatinib in this patient cohort. We present the following article in accordance with the TREND reporting checklist (available at http://dx.doi. org/10.21037/tcr-20-1773).

\section{Methods}

\section{Study design}

This was a prospective, phase II clinical trial conducted at the Zhejiang Cancer Hospital. The study protocol was approved by the ethics committee of the Zhejiang Cancer Hospital (NO: IRB-2017-126) and was performed in accordance with the Declaration of Helsinki (as revised in 2013) and Good Clinical Practice guidelines as defined by the International Conference on Harmonization. All patients provided written informed consent before taking part.

The primary endpoints were clinical benefit rate (CBR) and toxicity. CBR is defined as the percentage of patients achieving complete response, partial response, or stable disease according to the Response Evaluation Criteria In Solid Tumors (RECIST 1.1) criteria. Secondary endpoints included PFS and OS. The PFS was defined as time from the initiation of treatment to the date of disease progression or death from any causes, whichever came first; and OS was defined as time from the initiation of treatment to the time of death. We used Simon's twostage design, and analyzed the efficacy and toxicity in the intention-to-treat population (11). This study is registered with ClinicalTrials.gov, number NCT03213587.

\section{Patient selection}

Patients enrolled in this study were histologically or cytologically confirmed cases of recurrent or metastatic NPC who had failed at least one prior line of platinumbased chemotherapy for recurrent or metastatic disease. Other eligibility criteria were: at least one lesion accurately measured according to the Response Evaluation Criteria in Solid Tumors version 1.1 (RECIST 1.1); an Eastern Cooperative Oncology Group performance status (ECOG score) of 0 to 2 ; aged 18 to 65 years; adequate organ function (i.e., white blood cell count of $\geq 4.0 \times 10^{9} / \mathrm{L}$; absolute neutrophil count of $\geq 1.5 \times 10^{9} / \mathrm{L}$; hemoglobin concentrations of $\geq 90 \mathrm{~g} / \mathrm{L}$; platelet cell count of $\geq 100 \times 10^{9} / \mathrm{L}$; aspartate transaminase and alanine transaminase of $<2.5 \times$ the upper limit of the normal (ULN); if liver metastases, serum transaminase $<5 \times$ the ULN; and creatinine clearance rate of $\geq 60 \mathrm{~mL} / \mathrm{min}$ ); an estimated life expectancy of $\geq 3$ months; written informed consent; and amenable for regular follow-up.

Patients with any of the following conditions were 
excluded: prior therapy with a tyrosine kinase inhibitor targeting VEGFR or platelet-derived growth factor receptor (PDGFR); any factors that influence the usage of oral administration; known spinal cord compression or diseases of the brain or pia mater by CT/MRI screening; history of abdominal fistula, gastrointestinal perforation, or intra-abdominal abscess within 1 month of treatment, any history of cerebrovascular accident and artery or venous thromboembolic events within the last 6 months; any history of myocardial infarction, unstable angina pectoris, cardiac angioplasty or stent implantation within the last 3 months; blood coagulation abnormal with hemorrhagic tendency or thrombolysis or anticoagulation therapy. Patients with a preexisting serious accompanying disease that may put them at risk or influence their compliance, and those with secondary malignancies (except cured basal cell carcinoma of the skin and carcinoma in-situ of the uterine cervix) were excluded.

\section{Study treatment and assessment}

Patients received apatinib at an initial dose of $500 \mathrm{mg}$ once daily taken orally on an empty stomach in 28-day cycles and continued until disease progression, patient withdrawal, or unacceptable toxic effects. The protocoldefined dose modification criteria were as follows: when grade 3 hematological toxicity occurs in the patient, the drug should be suspended (for no longer than 2 weeks) and the original dose continued when the adverse reactions return to $\leq$ grade 2 ; if $\geq$ grade 3 adverse toxicity occurs again, the dose of apatinib should be reduced to $250 \mathrm{mg}$ (if grade 4 hematological toxicity occurs, the dose of apatinib should be immediately reduced to $250 \mathrm{mg}$ ); when grade 3 non-hematological toxicity occurs in the patient, the original dose should be continued after the adverse reaction is restored to $\leq$ grade 1 ; if $\geq$ grade 3 adverse reactions occur again, the dose of apatinib should be reduced to $250 \mathrm{mg}$.

Clinical, laboratory assessments, and QOL were performed at baseline and at one-month interval. Patients were provided with a blood pressure (BP) monitoring device and instructed to measure their BP once daily. Tumor assessments were performed at baseline and every month using RECIST 1.1 criteria. Objective responses were confirmed at least 4 weeks after initial documentation. Adverse events (AEs) were graded by the Common Terminology Criteria for Adverse Events (CTCAE v5.0). QOL was assessed by the European Organization for
Research and Treatment of Cancer (EORTC) Quality of Life Questionnaire-Core 30 (QLQ-C30) and the head and neck 35 module ( $\mathrm{H} \& \mathrm{~N} 35)$.

\section{Sample size estimation}

Simon's Minimax two-stage phase II design was used to calculate the sample size [13]. We considered the study drug to be inactive if CBR was $\leq 15 \%$, and considered the study drug as active if CBR was $\geq 35 \%$. Assuming the type I error of 0.05 and the type II error of 0.15 , the sample size and stopping rule were as follows: accrue 16 patients in stage I; if 3 or fewer of the 16 patients were nonprogressors, we would stop the study and conclude the study drug as inactive, otherwise proceed to stage II and accrue an additional 30 patients. After stage I interim analysis, the study proceeded to stage II and recruited a total of 19 patients but stopped for poor accrual.

\section{Statistical analysis}

We analyzed efficacy and safety in the intention-to-treat population. CBR was calculated with the corresponding exact $95 \%$ two-sided confidence interval (95\% CI) using standard methods based on the binomial distribution. Time-to-event data were summarized using the method of Kaplan-Meier. Paired Student's t-tests or Wilcoxon rank tests were used to compare QOL scores before and after treatment of apatinib. All statistical analyses were performed with SPSS18.0 (SPSS, Chicago, IL, USA). Results were considered statistically significant with a $\mathrm{P}$-value $<0.05$.

\section{Results}

\section{Patients characteristics}

Between Aug 2017 and Apr 2019, 19 patients were enrolled in this study (Table 1). All patients were ECOG 0-2, with a median age of 48 years (range, 23-64 years). Four (21.1\%) patients developed local-regional recurrent disease, 10 (52.6\%) had only distant metastasis and 5 (26.3\%) patients had both. The distant metastases were as follows: 5 (26.3\%) liver metastasis, 7 (36.8\%) lung metastasis, 6 (31.6\%) bone metastasis, 3 (15.8\%) distant lymph node metastasis, and 7 (36.8\%) multi-organ metastases. Eleven patients (57.9\%) patients had previously received one line of chemotherapy and seven $(36.8 \%)$ patients received second-line of 
Table 1 Baseline patient characteristics (N=19)

\begin{tabular}{|c|c|}
\hline Characteristic & Number of patients (\%) \\
\hline Age, years & 48 [23-64] \\
\hline \multicolumn{2}{|l|}{ Sex } \\
\hline Female & $4(21.1)$ \\
\hline Male & $15(78.9)$ \\
\hline \multicolumn{2}{|l|}{ ECOG performance status } \\
\hline 0 & $11(57.9)$ \\
\hline 1 & $7(36.8)$ \\
\hline 2 & $1(5.3)$ \\
\hline \multicolumn{2}{|l|}{ Metastasis number } \\
\hline 0 & $4(21.0)$ \\
\hline $1-2$ & $12(63.2)$ \\
\hline 3 & $3(15.8)$ \\
\hline \multicolumn{2}{|l|}{ Recurrent or metastasis sites } \\
\hline Recurrent disease & $4(21.1)$ \\
\hline Distant metastasis & $10(52.6)$ \\
\hline Recurrent and distant metastasis & $5(26.3)$ \\
\hline Liver & $5(26.3)$ \\
\hline Lung & $7(36.8)$ \\
\hline Bone & $6(31.6)$ \\
\hline Distant lymph nodes & $3(15.8)$ \\
\hline Multiple metastatic sites & $7(36.8)$ \\
\hline \multicolumn{2}{|l|}{$\begin{array}{l}\text { Previous lines of therapy for } \\
\text { recurrent/metastasis disease }\end{array}$} \\
\hline 1 & $11(57.9)$ \\
\hline 2 & $7(36.8)$ \\
\hline 3 or more & $1(5.3)$ \\
\hline
\end{tabular}

ECOG: Eastern Cooperative Oncology Group.

chemotherapy for the treatment of recurrent/metastatic disease.

\section{Treatment responses}

Tumor measurements were carried out monthly. There were no cases of complete response and two (10.5\%) patients showed a partial response to treatment. Eight $(42.1 \%)$ patients achieved stable disease, while six $(31.6 \%)$ had progressive disease. Three patients could not be evaluated for disease status because they had no post-baseline efficacy assessment. The CBR was 52.6\% (95\% CI: $29.8-76.2 \%$ ) at 3 months.

Four $(21.1 \%)$ patients received more than 6 months of treatment and the disease remained stable at 6 months. There was a reduction in tumor size in $42.1 \%$ of subjects at best response assessment (Figure 1). At the last followup visit, six $(31.6 \%)$ patients had disease progression and $12(63.2 \%)$ patients had died. The median PFS and OS were 3.7 (95\% CI: 0.6-6.8) months and 12.9 (95\% CI: 9.3-16.5) months, respectively (Figure 2). Subgroup analysis showed the $\mathrm{AE}$ of hand-foot syndrome was an independent prognostic factor for PFS [hazard ratio (HR) $=0.163,95 \%$ CI: $0.028-0.934, \mathrm{P}=0.042$ ).

\section{Toxicities}

Treatment-related AEs are listed in Table 2. Most patients developed grade 1-2 acute toxicities. In the intentionto-treat population, grade 3-4 AEs included: hand-foot syndrome [3 $(15.8 \%)]$, neutropenia [2 $(10.5 \%)]$, proteinuria [2 $(10.5 \%)]$, oral mucosal pain [2 $(10.5 \%)]$, hypertension [1 $(5.3 \%)]$, hyponatremia [1 (5.3\%)], artery dissection [1 $(5.3 \%)]$, and nasopharyngeal hemorrhage [1 (5.3\%)]. A serious $\mathrm{AE}$ was reported in one patient who died of nasopharyngeal hemorrhage. Six (31.6\%) patients required dose reductions from $500 \mathrm{mg}$ to $250 \mathrm{mg}$, which were related to proteinuria $(n=2)$, hand-foot syndrome $(n=2)$, epistaxis $(\mathrm{n}=1)$, and anorexia $(\mathrm{n}=1)$. Termination of medication occurred in two patients due to nasopharyngeal hemorrhage and arterial dissection. The median dose of apatinib was $357.9 \mathrm{mg}$ in this patient cohort.

\section{Quality of life}

The changes in patient-reported QOL before and after treatment are shown in Table 3. There was no clinically meaningful deterioration in QOL following treatment with apatinib as measured by the EORTC QLQ-C30 except for nausea \& vomiting (6.7 vs. 16.7, $\mathrm{P}=0.014$; Figure $3 A, B)$. Based on the EORTC QLQ-H\&N35, clinically meaningful worsening was seen in the domains of pain after treatment (pain: 6.1 vs. 23.4, $\mathrm{P}=0.001$; Figure $3 C$ ).

\section{Discussion}

The prognosis of patients with recurrent or metastatic NPC remains poor, with a median PFS of 4-10 months (12), and 


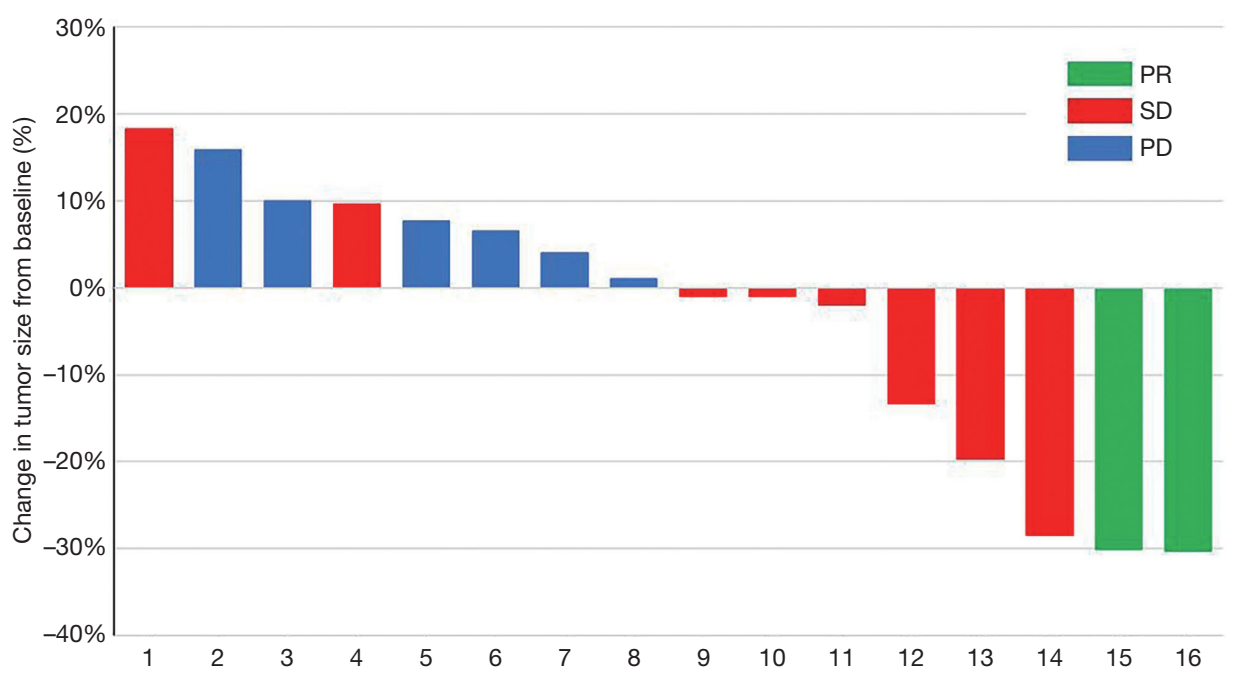

Figure 1 Waterfall plot for the best percentage change in target lesion size is shown for 16 patients who had at least one post-baseline efficacy assessment. The color indicates type of response. PR, partial response; SD, stable disease; PD, progressive disease.
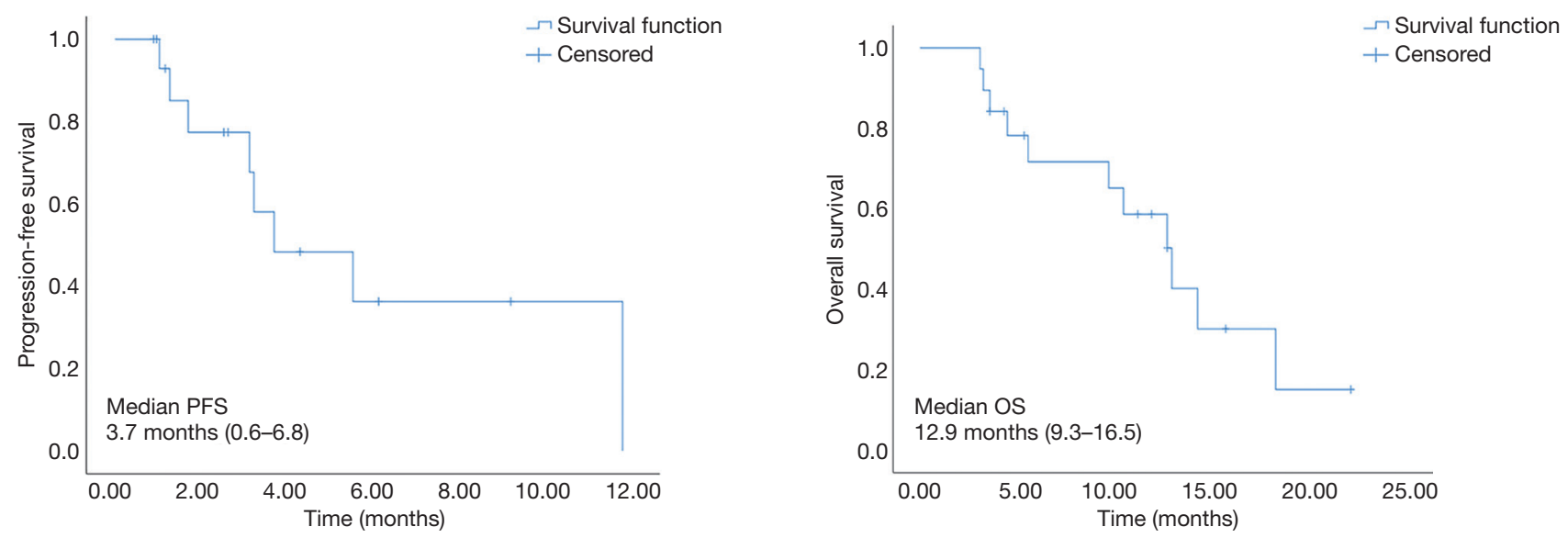

Figure 2 Kaplan-Meier survival curves of progression-free survival (PFS) and overall survival (OS).

there is currently no standard, effective treatment strategy for those who experience treatment failure after firstline platinum-based chemotherapy. The use of molecular targeted agents may be a promising strategy for this patient cohort. To the best of our knowledge, this is the first clinical study to evaluate the efficacy and safety of apatinib in treating patients with recurrent or metastatic NPC who failed prior platinum-based chemotherapy.

Several multi-kinase inhibitors (including sorafenib, pazopanib, axitinib, and sunitinib) have been investigated in recurrent or metastatic NPC (6-9). For example, pazopanib (an inhibitor of VEGFR-1, -2, and -3, PDGF-a, PDGF-b, and c-kit tyrosine kinase) was shown to have encouraging activity (CBR of $54.5 \%$ and median PFS of 4.4 months) in heavily pretreated patients with recurrent or metastatic NPC (9). In addition, dynamic-contrast enhanced computed tomography showed significant reductions in tumor blood flow with pazopanib treatment (7). Meanwhile, in another study, 13 patients with recurrent or metastatic NPC were treated with sunitinib monotherapy as a second-line chemotherapy, with modest results: the CBR was $28.6 \%$ and the median PFS was 3.5 months (9). Axitinib was also found to have modest disease control in NPC (6).

Apatinib is a highly-selective VEGFR-2 inhibitor, which has shown to be an effective treatment in advanced stomach cancer, colorectal cancer and non-small cell lung cancer 
Table 2 Treatment-related adverse events (according to CTCAE v5.0)

\begin{tabular}{|c|c|c|c|c|}
\hline & Grade $1-2, \mathrm{n}(\%)$ & Grade 3, n (\%) & Grade 4-5, n (\%) & All grades, $\mathrm{n}(\%)$ \\
\hline Hypertension & $8(42.1)$ & $1(5.3)$ & 0 & $9(47.4)$ \\
\hline Proteinuria & $10(52.6)$ & $2(10.5)$ & - & $12(63.2)$ \\
\hline Hand-foot syndrome & $7(36.28)$ & $3(15.8)$ & - & $10(52.6)$ \\
\hline Oral mucosal pain & $4(21.1)$ & $2(10.5)$ & 0 & $6(31.6)$ \\
\hline Nausea/Vomiting & $6(31.6)$ & 0 & 0 & $6(31.6)$ \\
\hline Anorexia & $10(52.6)$ & 0 & 0 & $10(52.6)$ \\
\hline Diarrhea & $6(31.6)$ & 0 & 0 & $6(31.6)$ \\
\hline Epistaxis & $5(26.3)$ & - & $1(5.3)$ & $6(31.6)$ \\
\hline APTT prolongation & $5(26.3)$ & 0 & - & $5(26.3)$ \\
\hline Weight loss & $3(15.8)$ & 0 & - & $3(15.8)$ \\
\hline Hypoalbuminemia & $5(26.3)$ & 0 & 0 & $5(26.3)$ \\
\hline Hyperbilirubinemia & $6(31.6)$ & 0 & 0 & $6(31.6)$ \\
\hline Elevated creatinine & $2(10.5)$ & 0 & 0 & $2(10.5)$ \\
\hline Elevated alanine aminotransferase & $4(21.1)$ & 0 & 0 & $4(21.1)$ \\
\hline Elevated aspartate aminotransferase & $8(42.1)$ & 0 & 0 & $8(42.1)$ \\
\hline Anemia & $10(52.6)$ & 0 & 0 & $10(52.6)$ \\
\hline Thrombocytopenia & $6(31.6)$ & 0 & 0 & $6(31.6)$ \\
\hline
\end{tabular}

APTT, activated partial thromboplastin time.

(13-15). In addition, apatinib was shown to inhibit tumor growth and have synergistic effects with chemotherapy in a nude mice model xenografted with CNE-2 NPC cells (10). In the present study, we observed apatinib had modest anticancer activity with a CBR of $52.6 \%$, a median PFS of 3.7 months, and a median OS of 12.9 months. Four (21.1\%) patients received more than 6 months of treatment and the disease remained stable at 6 months. Most patients had stable disease, which is consistent with its proposed antiangiogenesis mechanism of action. The efficiency of apatinib was comparable to other multi-kinase inhibitors mentioned above $(6-7,9)$.

Unfortunately, there was an unsatisfactory tolerance to apatinib, with $50 \%$ of patients requiring dose adjustment due to apatinib-related AEs. This resulted in a reduced dose of apatinib, which may have limited its overall efficacy. Indeed, our results suggest that apatinib at an initial dose of $500 \mathrm{mg}$ once daily taken orally may not be optimal for recurrent or metastatic NPC who failed prior cisplatin-based chemotherapy. Therefore, the optimal dose of apatinib and combined treatment mode should be explored in future. Notwithstanding, apatinib showed a relatively satisfactory 
Table 3 EORTC QLQ-C30 and H\&N35 scores comparison before and after treatment (mean \pm standard deviation)

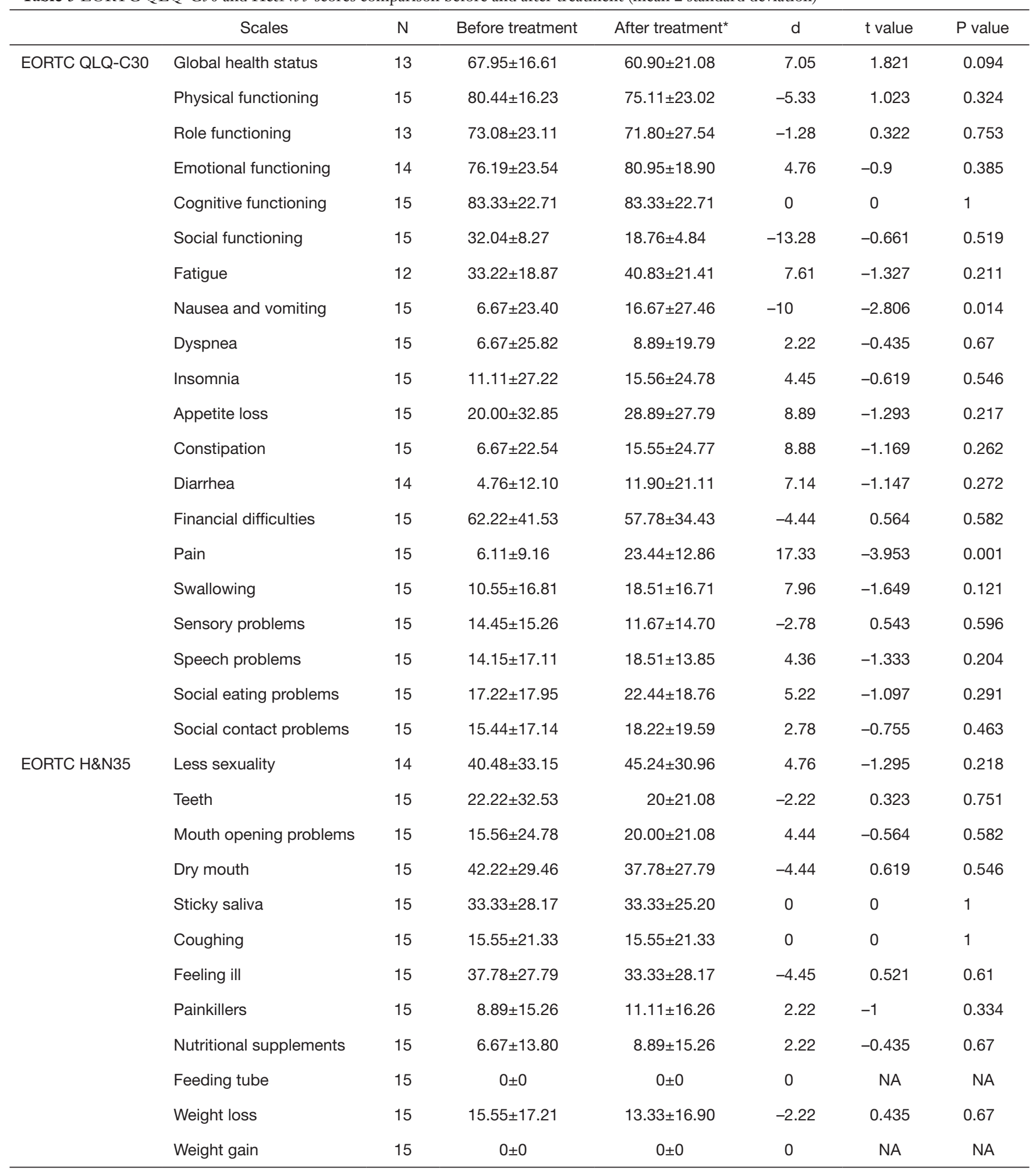

EORTC QOL-C30, European Organization for Research and Treatment of Cancer Quality of Life Questionnaire-Core 30; EORTC QOLH\&N35, The EOTRC Quality of Life Questionnaire-Head and Neck 35. NA, none. *, The score in the functional area after treatment is the highest score after the treatment cycle, and the score in the symptom area is the lowest score after the treatment cycle. 
A

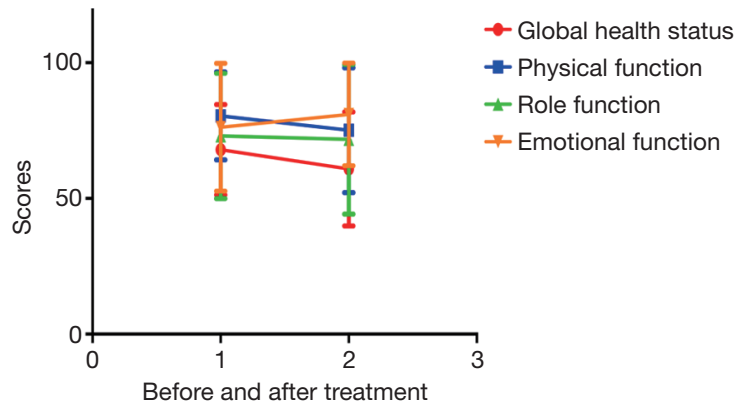

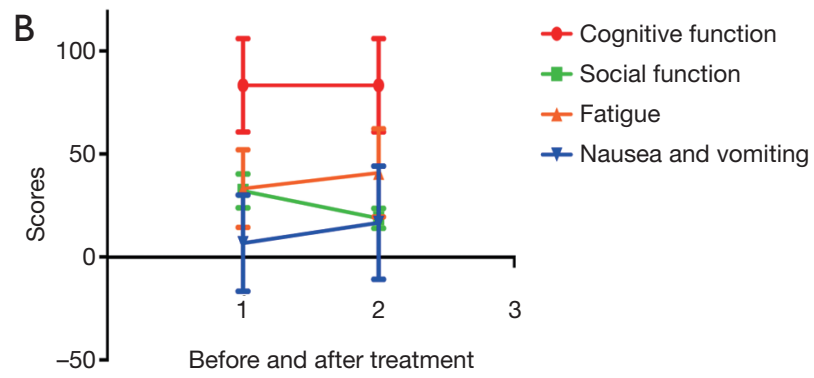

C

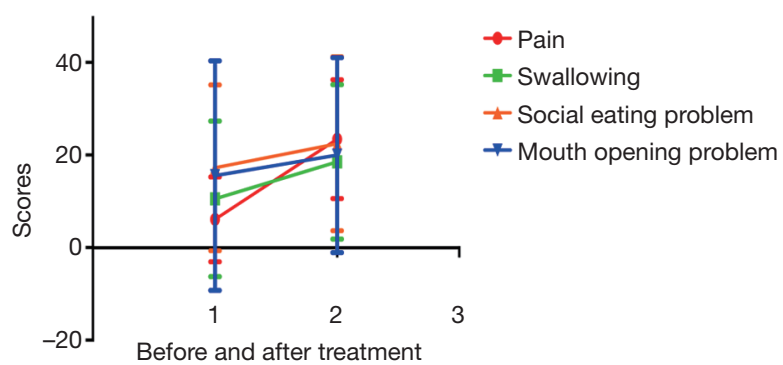

Figure 3 The QOL scores of patients before and after treatment of apatinib. (A) Adjusted mean change in global health status, physician function, role function and emotional function according to EORTC QLQ-C30. (B) Adjusted mean change in cognitive function, social function, fatigue and nausea \& vomiting according to EORTC QLQ-C30. (C) Adjusted mean change in pain, swallowing, social eating problem and month opening problem according to EORTC H\&N35. EORTC QOL-C30, European Organization for Research and Treatment of Cancer Quality of Life Questionnaire-Core 30; EORTC QOL-H\&N35, The EOTRC Quality of Life Questionnaire-Head and Neck 35; QOL, quality of life. *, $\mathrm{P}<0.05$.

anti-tumor activity and could be used as a therapeutic recommendation for recurrent or metastatic NPC.

Bleeding is one of the most common adverse effects of antiangiogenic agents. In our study, six (31.6\%) patients reported nasopharyngeal hemorrhage and one patient with recurrent NPC died. Admittedly, patients with recurrent NPC are prone to nasopharyngeal hemorrhage, potentially because these tumors are close to the internal carotid artery, and the ability to repair nasopharyngeal tissue after radiotherapy is poor. In our study, grade 3 to 4 hypertension, proteinuria and hand-foot syndrome occurred in $5.3 \%, 10.5 \%$ and $15.8 \%$ of patients, respectively. These toxicities of apatinib are similar to other multi-kinase inhibitors, including pazopanib and sunitinib $(7,9)$. In addition, the presence of apatinib-associated hypertension, proteinuria and hand-foot syndrome in the first 4 weeks of treatment was shown to be associated with prolonged median OS (16). Similarly, in the present study, patients with hand-foot syndrome showed better PFS than those without (median PFS: 5.5 vs. 1.7 months; $\mathrm{P}=0.042$ ). With regards to the patient-reported QOL outcomes, treatment with apatinib did not significantly influence the functional and symptom domains, except for nausea \& vomiting and pain (10 points or more deterioration, $\mathrm{P}<0.05)$. Therefore, the adverse effects of apatinib found in our study are considered moderate and acceptable when compared with historical reports of other antiangiogenic agents. Nonetheless, more attention should be paid to toxicity management when apatinib monotherapy or combined therapy is used in recurrent or metastatic NPC.

Whether combining antiangiogenic agents such as apatinib with other drugs improves their efficacy and reduces drug resistance is an area worth investigating. Preclinical studies have demonstrated that the use of antiangiogenic agents could convert the tumor microenvironment from an immunosuppressive one to one that is more immunosupportive (17). Indeed, combining low-dose apatinib treatment with anti-PD-L1 alleviated hypoxia, increased the infiltration of $\mathrm{CD} 8+\mathrm{T}$ cells and reduced recruitment of tumor-associated macrophages in a syngeneic lung cancer mouse model, resulting in significantly reduced tumor growth and prolonged survival (18). 
Furthermore, in a phase I clinical study, SH1210 (an anti-PD-1 antibody) combined with apatinib showed encouraging efficacy in refractory hepatocellular cancer with an overall response rate of 50\% (19). The anti-tumor activity of low-dose apatinib combined with anti-PD-1 in recurrent or metastatic NPC has also been observed in clinical practice. Therefore, the value of antiangiogenicbased combination therapy in NPC should be further investigated.

There are some limitations to our study. Due to the small sample size, these results should be interpreted with caution. In addition, due to the required dose reduction resulting from adverse effects, our study may not have revealed the maximal effect of this drug. Therefore, a larger clinical study is warranted to establish the optimal treatment mode of apatinib for recurrent or metastatic NPC.

\section{Conclusions}

Apatinib achieved modest disease control with acceptable toxicity in recurrent/metastatic NPC patients pretreated with platinum-based chemotherapy. A prospective, clinical trial is warranted to confirm the efficacy of apatinib in a large group of NPC patients, with a better defined dose schedule or as a combination therapy.

\section{Acknowledgments}

Funding: This work was supported by grants from Medical Health Science and Technology Project of Zhejiang Provincial Health Commission (No. 2020372435).

\section{Footnote}

Reporting Checklist: The authors have completed the TREND reporting checklist. Available at http://dx.doi. org/10.21037/tcr-20-1773

Data Sharing Statement: Available at http://dx.doi. org/10.21037/tcr-20-1773

Conflicts of Interest: All the authors have completed the ICMJE uniform disclosure form (available at http://dx.doi. org/10.21037/tcr-20-1773). The authors have no conflicts of interest to declare.

Ethical Statement: The authors are accountable for all aspects of the work in ensuring that questions related to the accuracy or integrity of any part of the work are appropriately investigated and resolved. The study was conducted in accordance with the Declaration of Helsinki (as revised in 2013). The study was approved by ethics committee of Zhejiang Cancer Hospital (NO.: IRB-2017126) and informed consent was taken from all the patients.

Open Access Statement: This is an Open Access article distributed in accordance with the Creative Commons Attribution-NonCommercial-NoDerivs 4.0 International License (CC BY-NC-ND 4.0), which permits the noncommercial replication and distribution of the article with the strict proviso that no changes or edits are made and the original work is properly cited (including links to both the formal publication through the relevant DOI and the license). See: https://creativecommons.org/licenses/by-nc-nd/4.0/.

\section{References}

1. Torre LA, Bray F, Siegel RL. Global cancer statistics 2012. CA Cancer J Clin 2015;65:87-108.

2. Her C. Nasopharyngeal cancer and the Southeast Asian patient. Am Fam Physician 2001;63:1776-82.

3. Jiang F, Jin T, Feng XL, et al. Long-term outcomes and failure patterns of patients with nasopharyngeal carcinoma staged by magnetic resonance imaging in intensitymodulated radiotherapy era: The Zhejiang Cancer Hospital's experience. J Cancer Res Ther 2015;11 Suppl 2:C179-84.

4. Lee AW, Sze WM, Au JS, et al. Treatment results for nasopharyngeal carcinoma in the modern era: the HongKong experience. Int J Radiat Oncol Biol Phys 2005;61:1107-16.

5. Zhang L, Huang Y, Hong S, et al. Gemcitabine plus cisplatin versus fluorouracil plus cisplatin in recurrent or metastatic nasopharyngeal carcinoma: a multicentre, randomised, open-label, phase 3 trial. Lancet 2016;388:1883-92.

6. Hui EP, Ma BBY, Loong HHF, et al. Efficacy, Safety, and Pharmacokinetics of Axitinib in Nasopharyngeal Carcinoma: A Preclinical and Phase II Correlative Study. Clin Cancer Res 2018;24:1030-7.

7. Lim WT, Ng QS, Ivy P, et al. A Phase II study of pazopanib in Asian patients with recurrent/metastatic nasopharyngeal carcinoma. Clin Cancer Res 2011;17:5481-9.

8. Elser C, Siu LL, Winquist E, et al. Phase II trial of sorafenib in patients with recurrent or metastatic squamous cell carcinoma of the head and neck or nasopharyngeal 
carcinoma. J Clin Oncol 2007;25:3766-73.

9. Hui EP, Ma BB, King AD, et al. Hemorrhagic complications in a phase II study of sunitinib in patients of nasopharyngeal carcinoma who has previously received high-dose radiation. Ann Oncol 2011;22:1280-7.

10. Peng QX, Han YW, Zhang YL, et al. Apatinib inhibits VEGFR-2 and angiogenesis in an in vivo murine model of nasopharyngeal carcinoma. Oncotarget 2017;8:52813-22.

11. Simon R. Optimal two-stage designs for phase II clinical trials. Control Clin Trials 1989;10:1-10.

12. Lee V, Kwong D, Leung TW, et al. Palliative systemic therapy for recurrent or metastatic nasopharyngeal carcinoma - How far have we achieved? Crit Rev Oncol Hematol 2017;114:13-23.

13. Jiang Q, Zhang NL, Ma DY, et al. Efficacy and safety of apatinib plus docetaxel as the second or above line treatment in advanced non squamous NSCLC: A multi center prospective study. Medicine 2019;98:e16065.

14. Liao X, Li H, Liu Z, et al. Clinical efficacy and safety of apatinib in patients with advanced colorectal cancer as the late-line treatment. Medicine 2018;97:e13635.

15. Li J, Qin S, Xu J, et al. Randomized, Double-Blind,

Cite this article as: Tao CJ, Zhang P, Zhou L, Hu QY, Chen $\mathrm{XZ}$. Apatinib in treating patients with recurrent or metastatic nasopharyngeal carcinoma who had failed prior platinum-based chemotherapy. Transl Cancer Res 2020;9(10):6392-6401. doi: $10.21037 /$ tcr-20-1773
Placebo-Controlled Phase III Trial of Apatinib in Patients With Chemotherapy-Refractory Advanced or Metastatic Adenocarcinoma of the Stomach or Gastroesophageal Junction. J Clin Oncol 2016;34:1448-54.

16. Liu X, Qin S, Wang Z, et al. Early presence of antiangiogenesis-related adverse events as a potential biomarker of antitumor efficacy in metastatic gastric cancer patients treated with apatinib: a cohort study. J Hematol Oncol 2017;10:153.

17. Fukumura D, Kloepper J, Amoozgar Z, et al. Enhancing cancer immunotherapy using antiangiogenics: opportunities and challenges. Nat Rev Clin Oncol 2018;15:325-40.

18. Zhao S, Ren S, Jiang T, et al. Low-Dose Apatinib Optimizes Tumor Microenvironment and Potentiates Antitumor Effect of PD-1/PD-L1 Blockade in Lung Cancer. Cancer Immunol Res 2019;7:630-43.

19. Xu J, Zhang Y, Jia R, et al. Anti-PD-1 Antibody SHR-1210 Combined with Apatinib for Advanced Hepatocellular Carcinoma, Gastric, or Esophagogastric Junction Cancer: An Open-label, Dose Escalation and Expansion Study. Clin Cancer Res 2019;25:515-23. 